\title{
Delay discounting in rhesus monkeys: Equivalent discounting of more and less preferred sucrose concentrations
}

\author{
Kevin B. Freeman • J. Emily Nonnemacher • \\ Leonard Green • Joel Myerson • William L. Woolverton
}

Published online: 27 August 2011

(C) Psychonomic Society, Inc. 2011

\begin{abstract}
Humans discount larger amounts of a delayed reinforcer less steeply than smaller amounts, but studies with pigeons and rats have yet to reveal such a magnitude effect, suggesting that the effect may be unique to humans. The present study examined whether the magnitude effect is observed in a species phylogenetically closer to humans, by comparing the rates at which rhesus monkeys discounted $10 \%$ and $20 \%$ concentrations of sucrose. There were no systematic differences in the rates at which the monkeys discounted the two sucrose concentrations, despite the fact that they strongly preferred the $20 \%$ concentration. Interestingly, the monkeys discounted delayed sucrose at a rate higher than was observed with delayed cocaine, and lower than was observed with delayed saccharin in previous studies (Freeman et al. Behavioural Processes, 82, 214-218, 2009; Woolverton et al. Experimental and Clinical Psychopharmacology, 15, 238-244, 2007). Taken together, these findings suggest that although both quantitative and qualitative differences can affect monkeys' preferences between immediate reinforcers, qualitative differences between types of reinforcers (e.g., sucrose vs. cocaine) can affect monkeys' discounting rates in a way that quantitative differences within a reinforcer (e.g., 10\% vs. $20 \%$ sucrose) do not.
\end{abstract}

K. B. Freeman $(\triangle) \cdot$ J. E. Nonnemacher $\cdot$ W. L. Woolverton Division of Neurobiology and Behavior Research,

Department of Psychiatry and Human Behavior,

University of Mississippi Medical Center,

Jackson, MS 39216, USA

e-mail: kfreeman@umc.edu

L. Green · J. Myerson

Department of Psychology, Washington University,

St. Louis, MO 63130, USA
Keywords Delay discounting $\cdot$ Magnitude effect . Hyperboloid discounting function $\cdot$ Sucrose $\cdot$ Monkeys

Delay discounting refers to the decrease in the value of a reinforcer as the time until its receipt increases. The relation between a reinforcer's subjective value and the delay to its receipt is generally well described by a hyperboloid discounting equation:

$V=A /(1+k D)$

where $V$ is the subjective value of the delayed reinforcer, $A$ is the amount of the delayed reinforcer, $k$ is a parameter that reflects the rate of discounting, and $D$ is the amount of time until the receipt of the delayed reinforcer. Equation 1 provides an accurate description of discounting rates for a variety of reinforcers in both human and nonhuman subjects, although human delay discounting data are often even better described by a generalized form of Eq. 1, in which the denominator is raised to a power. Subjective value is frequently expressed as a proportion or percentage of the amount of the delayed reinforcer (i.e., as relative subjective value) to facilitate comparisons between different delayed amounts. Hyperboloid discounting functions have been used to compare discounting of reinforcers both within and across species (for a review, see Green \& Myerson, 2004).

An important finding in studies of human discounting is the relation between reinforcer magnitude and discounting rate: Humans discount larger amounts of a delayed reinforcer less steeply than smaller amounts, a finding referred to as the magnitude effect (Green, Myerson, \& McFadden, 1997; Kirby, 1997; Raineri \& Rachlin, 1993). In contrast, varying the magnitude of delayed reinforcers in studies with rats and pigeons, the only nonhuman species 
that have been rigorously tested for magnitude effects, has yet to provide evidence of a magnitude effect in animals, regardless of whether the magnitude of delayed reinforcers is varied by manipulating their amount or their quality (Calvert, Green, \& Myerson, 2010; Green, Myerson, Holt, Slevin, \& Estle, 2004; Richards, Mitchell, de Wit, \& Seiden, 1997).

Although studies with humans typically use hypothetical reinforcers, whereas studies with nonhumans have exclusively used actual reinforcers, the lack of a magnitude effect in rats and pigeons is not likely due to differences between the discounting of hypothetical and actual reinforcers. This is because magnitude effects have been reported in humans discounting actual money (Johnson \& Bickel, 2002; Kirby, 1997), as well as liquid reinforcers (Jimura, Myerson, Hilgard, Braver, \& Green, 2009). The pattern of results suggests that only humans discount delayed reinforcers of varying magnitudes at different rates. However, the effect of reinforcer magnitude on discounting by animals closer in phylogeny to humans (e.g., monkeys) obviously needs to be considered before accepting this conclusion.

Woolverton, Myerson, and Green (2007) reported delay discounting in rhesus monkeys using intravenous cocaine as the reinforcer, and their discounting was well described by a hyperbolic function (Eq. 1). Subsequently, Freeman, Green, Myerson, and Woolverton (2009), using some of the same monkeys studied by Woolverton et al., examined delay discounting using saccharin as the reinforcer, and they found that these monkeys discounted saccharin more steeply than they had discounted cocaine.

One interesting possibility raised by the Woolverton et al. (2007) and Freeman et al. (2009) studies is that the monkeys discounted cocaine less steeply than saccharin because cocaine was more highly valued, and that this finding represents a magnitude effect analogous to the finding that humans discount larger amounts of money less steeply than smaller amounts. However, Freeman et al. also included a limited assessment of the magnitude effect by comparing the discounting of two amounts of delayed saccharin solution at a single delay, and they found no systematic difference in the degrees to which the two magnitudes were discounted. Nevertheless, further research that contrasts complete discounting functions for different reinforcer magnitudes is needed before conclusions can be drawn about the effect of reinforcer magnitude on discounting rates in monkeys.

In addition, it may be important to go beyond the use of the amount of immediate reinforcer as the measure of subjective value, particularly in nonhuman animals. Behavioral economics assumes that the value of different goods/ reinforcers is reducible to a single currency or dimensionutility - and in humans, amount is typically assumed to map directly onto that dimension. However, other character- istics of reinforcers also may be determinants of reinforcer value in animals, and it is of interest whether such characteristics will map reliably onto utility in nonhuman animals, and perhaps especially whether they do so in nonhuman primates. Two issues are involved here: One is whether manipulating characteristics of delayed reinforcers other than their amount in such a way as to alter their value will result in a magnitude effect (Calvert et al., 2010; Farrar, Kieres, Hausknecht, de Wit, \& Richards, 2003). The other is whether the subjective value of a delayed reinforcer can be assessed by manipulating the characteristics of an immediate reinforcer other than its amount until the immediate and delayed reinforcers are equally likely to be chosen (Woolverton et al., 2007).

Accordingly, the purpose of the present study was to compare the discounting functions for two delayed sucrose reinforcers that varied in sweetness, using the concentration of the immediate reinforcer to assess subjective value in nonhuman primates. Specifically, rhesus monkeys were allowed to choose between immediate and delayed 2-ml solutions of sucrose. In separate conditions, the concentration of the delayed reinforcer was either $10 \%$ or $20 \%$. The concentration of the immediate sucrose solution was also varied in order to establish the immediate sucrose concentration that was equal in subjective value to the delayed reinforcer, and this process was repeated at different delays in order to map out separate discounting functions for the two delayed sucrose concentrations. At issue was (1) whether a hyperbolic function (Eq. 1) would describe discounting when the subjective value of a delayed reinforcer was measured in terms of the concentration of an immediate sucrose reinforcer, and (2) whether monkeys would show a magnitude effect by discounting a more highly valued $(20 \%)$ delayed sucrose solution less steeply than a lower-valued (10\%) sucrose solution.

\section{Method}

Subjects

A total of 5 male rhesus monkeys (Macaca mulatta) weighing between 9.25 and $11.50 \mathrm{~kg}$ served as subjects. Of the monkeys, 4 (BDH, DCJ, R0105, and X4F) had histories of discounting delayed saccharin reinforcers (Freeman et al., 2009), and 2 (DCJ and R0105) also had a history of discounting delayed cocaine reinforcers (Woolverton et al., 2007). Another monkey (M163) had previous experience in drug discrimination with $d$-amphetamine (most recently, Fantegrossi, Woolverton, Winger, Coop, \& Woods, 2005). Approximately $4 \mathrm{~h}$ before the start of an experimental session, each monkey was fed a sufficient amount of monkey chow (Teklad 25\% Monkey Diet; Harlan/Teklad, Madison, 
WI) to maintain stable body weight. Each monkey was given fresh fruit daily and a chewable vitamin tablet 3 days a week. Water was available ad libitum. Lighting was cycled to maintain $16 \mathrm{~h}$ of light and $8 \mathrm{~h}$ of darkness, with lights on at 6:00 a.m.

\section{Apparatus}

Each monkey was fitted with either a mesh jacket (Lomir Biomedical, Malone, NY) or a stainless-steel restraint harness (E\&H Engineering, Chicago, IL) and tether that attached to the rear of a $1.0-\mathrm{m}^{3}$ experimental cubicle (PlasLabs, Lansing, MI), in which the monkey lived throughout the experiment. Exhaust fans mounted on the back of each cubicle provided ventilation and masking noise. There were two response levers (PRL-001; BRS/LVE, Beltsville, MD) mounted in steel boxes on the interior of the transparent polycarbonate front wall of each experimental cubicle. The response levers were located $10.0 \mathrm{~cm}$ above the floor and $47.0 \mathrm{~cm}$ apart. Four jeweled stimulus lights, two white and two red, were located directly above each lever. Variablespeed injection pumps (7540X; Cole-Parmer, Chicago, IL) located outside the cubicle delivered sucrose solution through plastic tubing to metal sipper tubes positioned $10.0 \mathrm{~cm}$ above the lever boxes. Each 2-ml reinforcer, whether immediate or delayed, was delivered over a 10 -s interval, and pumps were calibrated weekly to ensure stable volume output. Programming and recording of experimental events were recorded using a Macintosh computer and the associated interfaces.

\section{Procedure}

Sessions began daily at approximately 11:00 a.m. Each session consisted of 64 trials, the first 4 of which were forced choice (sampling) trials (2 for each leverreinforcer pairing), followed by 60 free choice trials. During forced choice trials, only one of the two levers was active, and the active lever, signaled by illumination of its pair of white lights, alternated in order to ensure equal exposure to the contingencies programmed for each lever. During free choice trials, both pairs of white lever lights were illuminated, and both levers were active. On all trials, a single leverpress extinguished the white lights, illuminated the red lights, and initiated a 120 -s intertrial interval (ITI). If the monkey pressed the lever associated with the immediate reinforcer, sucrose was delivered immediately to the spout for $10 \mathrm{~s}$, after which the red lights were extinguished. If the monkey pressed the lever associated with the delayed reinforcer, the red lights flashed once per second for the duration of the delay and then remained continuously illuminated during the 10 -s reinforcer delivery. The ITI and the delay ran simultaneously, ensuring that reinforcement rate did not covary with delay to reinforcement.

The delayed reinforcer was $2.0 \mathrm{ml}$ of either $10 \%$ or $20 \%$ sucrose solution presented after a delay that ranged from 0 to $110 \mathrm{~s}$. The immediate reinforcer was also $2.0 \mathrm{ml}$ of sucrose solution, the concentration of which ranged from $1.2 \%$ to $28 \%$. For 4 of the monkeys (BDH, DCJ, R0105, and $\mathrm{X} 4 \mathrm{~F}$ ), discounting functions for the two magnitudes of delayed sucrose were completed in an ABA design, such that the $20 \%$ delayed sucrose concentration was tested first, followed by $10 \%$ sucrose, and ending with a redetermination of $20 \%$ sucrose. For the remaining monkey (M163), the $10 \%$ delayed sucrose concentration was tested first, followed by $20 \%$ sucrose, and ending with a $10 \%$ sucrose redetermination.

Within each delayed magnitude condition, delays were studied in an irregular order both within and between monkeys, and the range of delays varied across monkeys. A concentration-response function at a particular delay (as shown in Fig. 1) was completed before moving on to another delay. Furthermore, within each delay condition, at least three immediate concentrations were tested in an irregular order. If none of the three immediate concentrations resulted in stable preference between $20 \%$ and $80 \%$, at least one additional immediate concentration was then tested in order to obtain at least two for which preference was less than $20 \%$ and two for which it was greater than $80 \%$.

In each session, the 60 free choice trials were divided into three 20-trial blocks. A given condition was in effect for at least 2 days and until choice was stable across all three blocks within a session. Stability was defined as follows: (a) The number of choices of the immediate reinforcer for each block was within $20 \%$ of the three-block mean, and (b) there was no upward or downward trend across blocks. Once choice was stable, the reinforcer-lever pairing was reversed, and stable preference was redetermined. Typically, if choice averaged about $50 \%$, this reflected a strong position preference that was maintained across the reinforcer-lever reversal rather than indicating indifference between the two reinforcers (i.e., equal choice of the two alternatives in both reversal conditions).

\section{Data analysis}

For each pair of lever reversal conditions run at a specific delay and delayed sucrose concentration, the mean number of choices of the immediate sucrose concentration at stability was converted to a percentage of total choices and used as the measure of preference. Concentration-response functions were determined at each delay, and the indifference point (i. e., the concentration of the immediate reinforcer predicted to 
A

B

A

$\mathrm{BDH}$

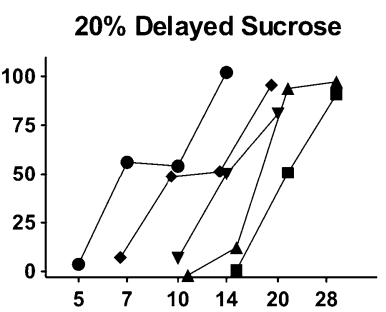

$10 \%$ Delayed Sucrose

$20 \%$ Delayed Sucrose

DCJ
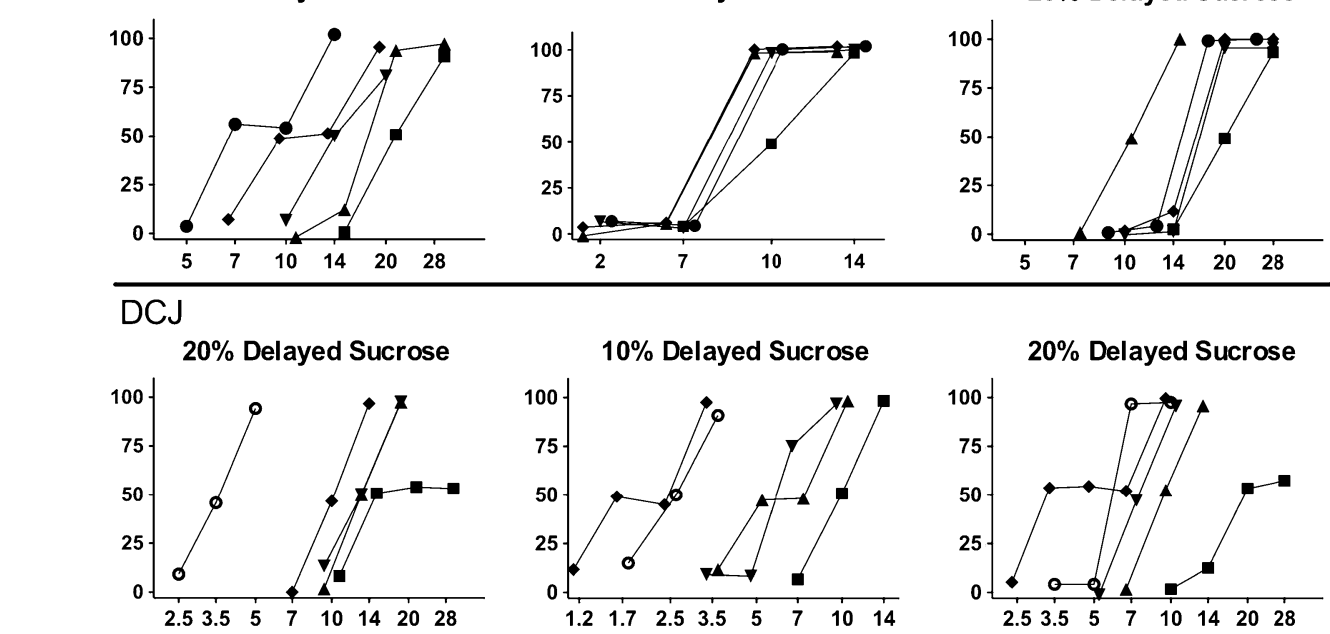

$20 \%$ Delayed Sucrose
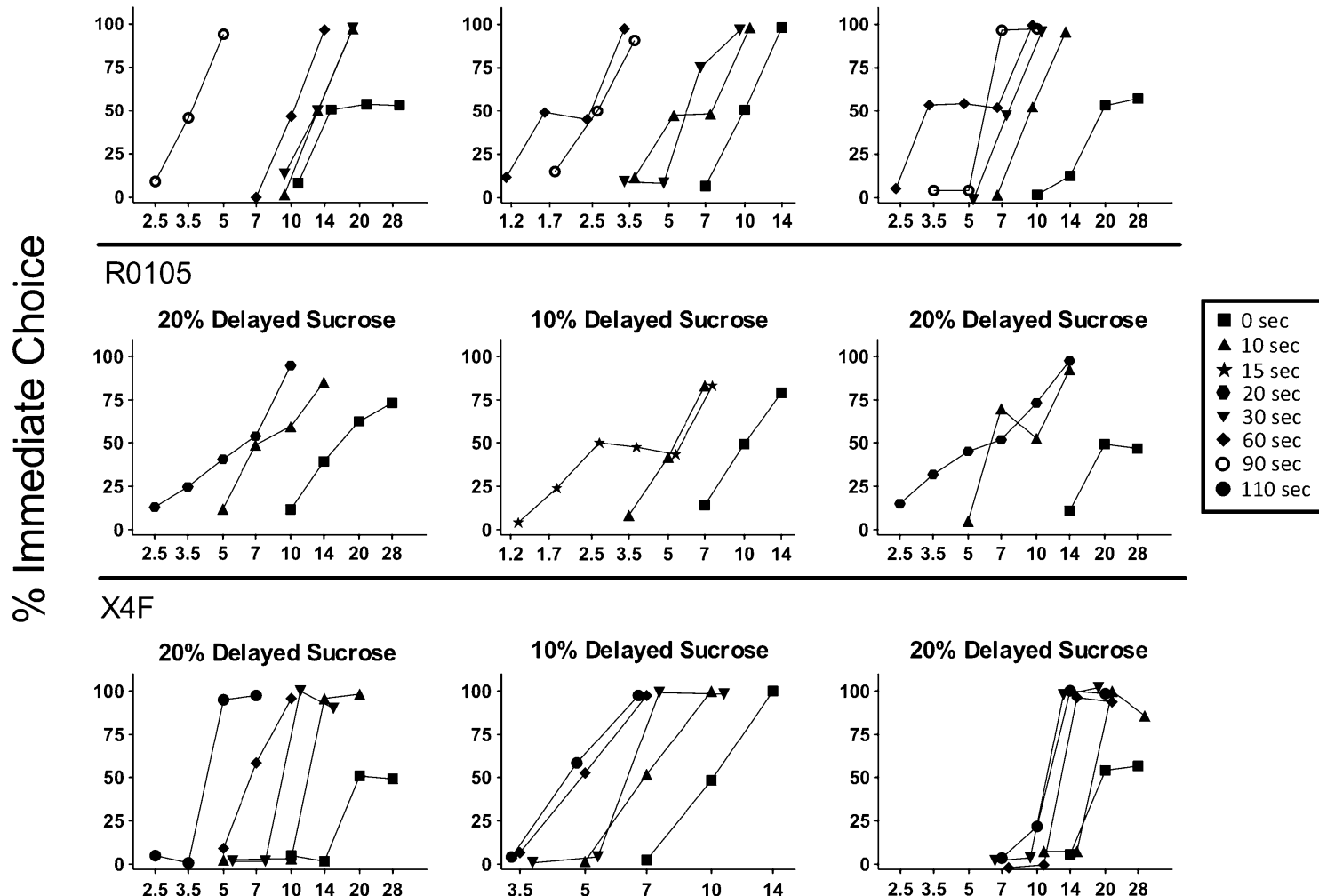

\section{M163}
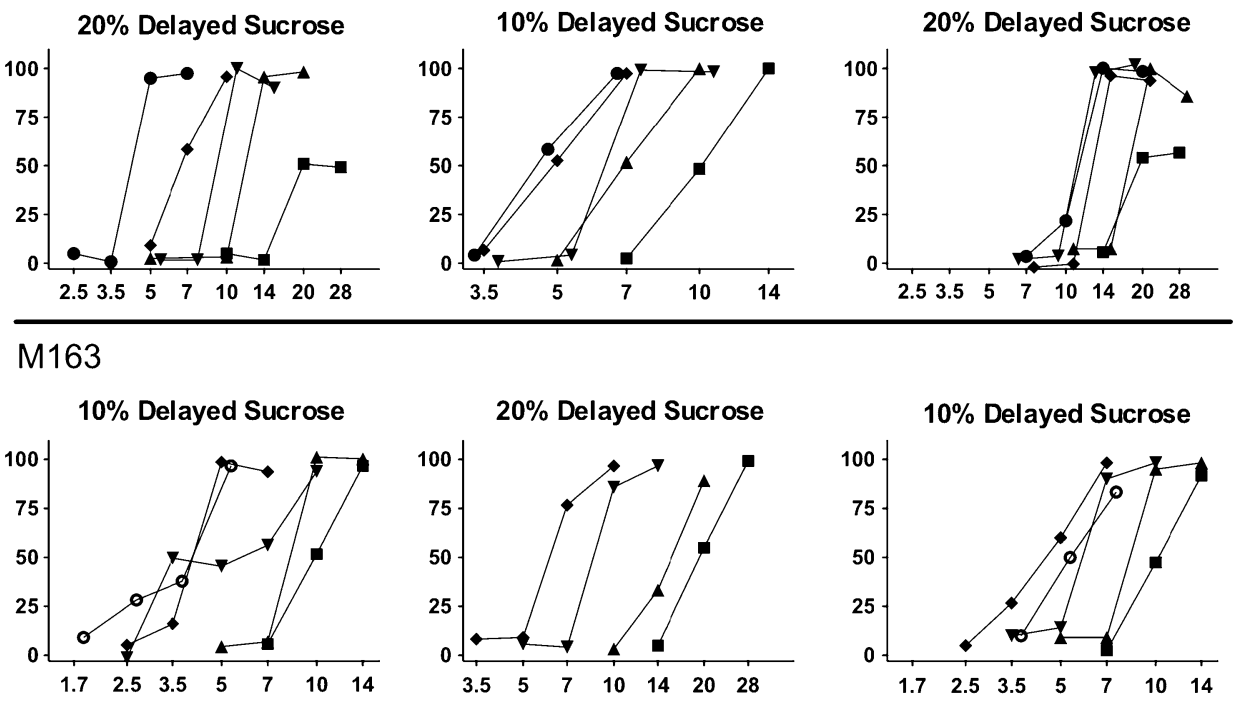

Sucrose Concentration (\%)

Fig. 1 Percentage choice of immediate sucrose as a function of concentration. The concentration of the delayed reinforcer was either $10 \%$ or $20 \%$ sucrose. "A B A" refers to the order in which the delayed sucrose concentrations were studied in each subject

result in approximately equal choices of the immediate and delayed reinforcers) was calculated on the basis of the fits of a logistic concentration-response function (GraphPad 5.0). Fits of the logistic function were for the most part very good (mean $R^{2}=.92$, median $=.99$ ). The indifference point, expressed as a percentage of the concentration of the delayed reinforcer, 
represents the delayed reinforcer's relative subjective value. Finally, relative subjective value was plotted as a function of delay and fitted with Eq. 1, the hyperbolic discounting function.

\section{Results}

As may be seen in Fig. 1, choice for the immediate reinforcer typically increased as a function of its concentration in all monkeys at each delay. Of particular significance are the data for the 0-s delay conditions in which monkeys chose between two immediate 2-ml sucrose reinforcers of different concentrations. Note that the 3 monkeys (DCJ, X4F, and R0105) who were given a choice between an immediate $20 \%$ sucrose reinforcer and an immediate $10 \%$ sucrose reinforcer all preferred the higher concentration, and the other 2 monkeys (BDH and M163), who were given a choice between immediate $20 \%$ and $14 \%$ sucrose reinforcers, also preferred the higher concentration. Thus, over the range examined in the present study, subjective value was an increasing function of concentration.

As also may be seen in Fig. 1, the concentrationresponse functions for sucrose (i.e., percentage choice of the immediate reinforcer as a function of its concentration) tended to shift systematically to the left in each monkey as the time until the delayed reinforcer increased. This shift reflects the fact that as the delay increased, progressively lower concentrations of immediate sucrose were preferred to the delayed higher concentration. An exception was subject $\mathrm{BDH}$ in the delayed $10 \%$ sucrose condition, for whom increasing the time until the delayed reinforcer beyond $10 \mathrm{~s}$ had little additional effect on choice between sucrose concentrations.

Figure 2 depicts the relative subjective value of the 10\% and $20 \%$ sucrose reinforcers as a function of delay for each monkey. Where initial values were redetermined, the average of the two determinations is presented. Recall that subjective value equals the concentration of immediate sucrose reinforcer equivalent in value to the delayed reinforcer, and that subjective value is expressed as a percentage of the concentration of the delayed reinforcer (i.e., as relative subjective value), in order to facilitate comparison of the discounting of the $10 \%$ and $20 \%$ sucrose concentrations.

As may be seen, relative subjective value decreased systematically as a function of delay for 4 of the 5 monkeys (the exception again being $\mathrm{BDH}$ ), and the hyperbolic discounting function, Eq. 1, provided a good fit to their data (all $R^{2} \mathrm{~s}$ were at least .70 for both delayed reinforcers; see Table 1). For these 4 monkeys, the $k$ parameter in Eq. 1 is an appropriate measure of how steeply they discounted.

Note that 3 of these monkeys were studied twice with the delayed $20 \%$ sucrose reinforcer, and there was no systematic difference between the initial and redetermined values of their $k$ parameters: In one case (DCJ), $k$ increased, in one case it decreased (X4F), and in the third case (R0105), it did not change (see Table 1). Therefore, we averaged initial and redetermined $k$ values for the purpose of comparing the discounting of delayed $10 \%$ and $20 \%$ sucrose reinforcers. A Wilcoxon signed rank test failed to reveal steeper discounting of the $10 \%$ sucrose concentration, $Z=1.826, p=.125$. Although such a test is underpowered, we would point out that the observed difference is in the direction opposite to what would be expected if there were a magnitude effect (i. e., if higher-valued reinforcers were discounted less steeply): For the $10 \%$ concentration, the mean $k$ was 0.04 , which indicates shallower discounting than that observed for the more highly valued $20 \%$ concentration, for which the mean $k$ was 0.06 .

An additional measure of discounting is provided by the area under the empirical discounting curve, or $\mathrm{AuC}$ (Myerson, Green, \& Warusawitharana, 2001), which for present purposes has the advantage of not relying on how well a particular theoretical model (e.g., the hyperbolic discounting function) describes the data. The AuC can range between 0.0 (steepest possible discounting) and 1.0 (no discounting of the delayed reinforcer). Averaged across the 5 monkeys, the mean $\mathrm{AuC}$ was .610 for the delayed $10 \%$ sucrose concentration and .594 for the delayed $20 \%$ sucrose concentration $(t<1.0)$, indicating equivalent discounting of the two concentrations.

\section{Discussion}

Increasing the delay of a sucrose reinforcer systematically decreased its value across monkeys, and for 4 of the 5 monkeys this decrease was well described by the hyperbolic discounting function (Eq. 1). Despite the fact that $20 \%$ sucrose was preferred to a lower sucrose concentration by all of the monkeys, delayed $20 \%$ sucrose reinforcers were not discounted less steeply than delayed $10 \%$ sucrose reinforcers, consistent with the results of our previous study (Freeman et al., 2009), which failed to find evidence of a magnitude effect in monkeys using delayed saccharin reinforcers.

The lack of a magnitude effect when reinforcer amount or concentration was varied stands in contrast to the finding that monkeys discount different types of reinforcers at different rates. We previously reported that monkeys who were subjects in both the Woolverton et al. (2007) and Freeman et al. (2009) studies discounted delayed saccharin reinforcers more steeply than delayed cocaine reinforcers. We also note that the 2 monkeys (DCJ and R0105) who were in both Woolverton et al. and the present study discounted sucrose more steeply than cocaine, and the 4 monkeys (BDH, DCJ, R0105, and X4F) who were in both Freeman et al. and the present study all 
Fig. 2 Relative subjective value of $10 \%$ and $20 \%$ sucrose reinforcers as a function of delay. The curves represent the bestfitting hyperbolic discounting functions (Eq. 1). Value is expressed as the percentage of the concentration of the delayed sucrose reinforcer. Redetermination conditions were averaged within each subject

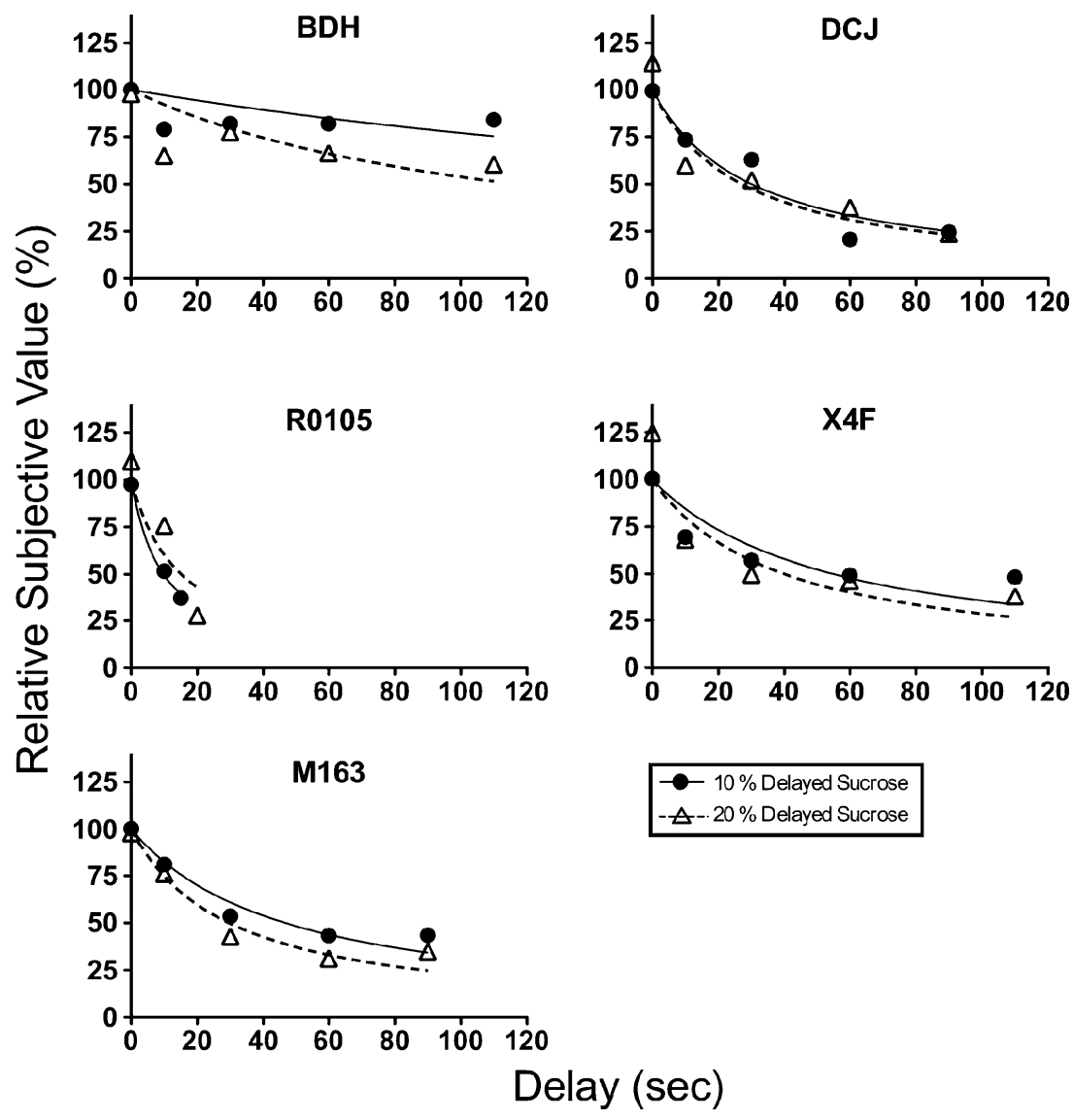

discounted saccharin more steeply than sucrose. Averaged across all of the monkeys in each study, the mean $k$ values were 0.02 for delayed cocaine, 0.05 for delayed sucrose, and 0.12 for delayed saccharin. Thus, it appears that saccharin is discounted more steeply than sucrose, which in turn is discounted more steeply than cocaine.

Notably, the degree to which these three reinforcers were discounted is inversely related to their order of preference as determined in choice tests with 2 monkeys who, like those in the present study, were not deprived (unpublished data). These
2 monkeys, 1 of which was used in the present study (X4F), preferred sucrose at or below a concentration of $10 \%$ to $0.05 \%$ saccharin (the concentration used in Freeman et al., 2009). Furthermore, another monkey, given a choice between intravenous cocaine and $20 \%$ sucrose, preferred cocaine at a dose as low as $0.025 \mathrm{mg} / \mathrm{kg}$ (lower than that of the delayed cocaine reinforcer used in Woolverton et al., 2007). Thus, the most preferred reinforcer, cocaine, was discounted at the lowest rate, and the least preferred reinforcer, saccharin, was discounted at the highest rate.
Table 1 Individual $R^{2}$ and $k$ values for delayed $10 \%$ and $20 \%$ sucrose concentrations

"n.r." indicates that a condition was not run. $R^{2}=.000$ indicates that the variance in the residuals for Eq. 1 was greater than the variance about the mean.

\begin{tabular}{|c|c|c|c|c|c|}
\hline & & \multicolumn{2}{|c|}{$10 \%$ Delayed Sucrose } & \multicolumn{2}{|c|}{$20 \%$ Delayed Sucrose } \\
\hline & & Initial & Redetermination & Initial & Redetermination \\
\hline \multirow[t]{2}{*}{$\mathrm{BDH}$} & $k$ & 0.003 & n.r. & 0.014 & 0.004 \\
\hline & $R^{2}$ & .000 & n.r. & .955 & .000 \\
\hline \multirow[t]{2}{*}{ DCJ } & $k$ & 0.033 & n.r. & 0.024 & 0.064 \\
\hline & $R^{2}$ & .926 & n.r. & .850 & .849 \\
\hline \multirow[t]{2}{*}{ R0105 } & $k$ & 0.102 & n.r. & 0.149 & 0.149 \\
\hline & $R^{2}$ & .992 & n.r. & .920 & .842 \\
\hline \multirow[t]{2}{*}{$\mathrm{X} 4 \mathrm{~F}$} & $k$ & 0.018 & n.r. & 0.048 & 0.013 \\
\hline & $R^{2}$ & .727 & n.r. & .876 & .700 \\
\hline \multirow[t]{2}{*}{ M163 } & $k$ & 0.025 & 0.018 & 0.034 & n.r. \\
\hline & $R^{2}$ & .954 & .926 & .953 & n.r. \\
\hline
\end{tabular}


Although these findings will need to be replicated in a study that compares discounting rates and preference among different types of reinforcers in the same monkeys, the pattern of results suggests that qualitative differences between types of reinforcers may affect monkeys' discounting rates in a way that seems analogous to the magnitude effect observed in humans (i.e., shallower discounting of more highly valued rewards), even though quantitative differences within a type of reinforcer do not. More work will be needed to determine the extent to which these findings generalize to other drug and nondrug reinforcers, as well as to other animals. For example, rats appear to discount qualitatively different reinforcers, including both different foods and different liquids, at comparable rates, even when there are clear differences in preference between foods as well as between liquids (Calvert et al., 2010), raising the possibility that primates may differ from nonprimates in this regard.

In addition to the question of whether monkeys show a magnitude effect, the other important issue addressed by the present study was whether a hyperbolic function (Eq. 1) would describe discounting when the subjective value of a delayed reinforcer was measured in terms of the concentration of the immediate reinforcer, rather than in terms of the amount of immediate reinforcer. For 4 out of the 5 monkeys, a hyperbolic discounting function provided a good description of their data at both delayed amounts. The significance of this finding becomes clear when considered in the context of previous results showing hyperbolic discounting when subjective value was measured in terms of amount (i.e., milliliters of saccharin; Freeman et al., 2009) or dose (i.e., milligrams/kilogram of cocaine; Woolverton et al., 2007). Taken together with these previous findings, the present results showing hyperbolic discounting when subjective value was measured in terms of sweetness (i.e., percent sucrose concentration) are consistent with the idea that a single underlying dimension (value or utility) can be measured in different ways and that this dimension captures the desirability of a variety of different types of reinforcers, thereby providing a common basis for monkeys' decisions involving delayed outcomes.

Author note This research was supported by the Drug Abuse Research and Development Fund, University of Mississippi Medical Center. In addition, manuscript preparation was supported in part by National Institutes of Health Grant MH055308 to L.G. and J.M. The authors gratefully acknowledge the technical assistance of Brian McMaster, Lindsey Halley, and Jeremy Wilson.

\section{References}

Calvert, A. L., Green, L., \& Myerson, J. (2010). Delay discounting of qualitatively different reinforcers in rats. Journal of the Experimental Analysis of Behavior, 93, 171-184. doi:10.1901/ jeab.2010.93-171

Fantegrossi, W. E., Winger, G., Woods, J. H., Woolverton, W. L., \& Coop, A. (2005). Reinforcing and discriminative stimulus effects of 1-benzylpiperazine and trifluoromethylphenylpiperazine in rhesus monkeys. Drug and Alcohol Dependence, 77, 161-168. doi:10.1016/j.drugalcdep.2004.07.014

Farrar, A. M., Kieres, A. K., Hausknecht, K. A., de Wit, H., \& Richards, J. B. (2003). Effects of reinforcer magnitude on an animal model of impulsive behavior. Behavioural Processes, 64, 261-271. doi:10.1016/S0376-6357(03)00139-6

Freeman, K. B., Green, L., Myerson, J., \& Woolverton, W. L. (2009). Delay discounting of saccharin in rhesus monkeys. Behavioural Processes, 82, 214-218. doi:10.1016/j.beproc.2009.06.002

Green, L., \& Myerson, J. (2004). A discounting framework for choice with delayed and probabilistic rewards. Psychological Bulletin, 130, 769-792. doi:10.1037/0033-2909.130.5.769

Green, L., Myerson, J., \& McFadden, E. (1997). Rate of temporal discounting decreases with amount of reward. Memory \& Cognition, 25, 715-723. doi:10.3758/BF03211314

Green, L., Myerson, J., Holt, D. D., Slevin, J. R., \& Estle, S. J. (2004). Discounting of delayed food rewards in pigeons and rats: Is there a magnitude effect? Journal of the Experimental Analysis of Behavior, 81, 39-50. doi:10.1901/jeab.2004.81-39

Jimura, K., Myerson, J., Hilgard, J., Braver, T. S., \& Green, L. (2009). Are people really more patient than other animals? Evidence from human discounting of real liquid rewards. Psychonomic Bulletin \& Review, 16, 1071-1075. doi:10.3758/PBR.16.6.1071

Johnson, M. W., \& Bickel, W. K. (2002). Within-subject comparison of real and hypothetical money rewards in delay discounting. Journal of the Experimental Analysis of Behavior, 77, 129-146. doi:10.1901/jeab.2002.77-129

Kirby, K. N. (1997). Bidding on the future: Evidence against normative discounting of delayed rewards. Journal of Experimental Psychology. General, 126, 54-70. doi:10.1037/00963445.126.1.54

Myerson, J., Green, L., \& Warusawitharana, M. (2001). Area under the curve as a measure of discounting. Journal of the Experimental Analysis of Behavior, 76, 235-243. doi:10.1901/ jeab.2001.76-235

Raineri, A., \& Rachlin, H. (1993). The effect of temporal constraints on the value of money and other commodities. Journal of Behavioral Decision Making, 6, 77-94. doi:10.1002/ bdm.3960060202

Richards, J. B., Mitchell, S. H., de Wit, H., \& Seiden, L. S. (1997). Determination of discount functions in rats with an adjustingamount procedure. Journal of the Experimental Analysis of Behavior, 67, 353-366. doi:10.1901/jeab.1997.67-353

Woolverton, W. L., Myerson, J., \& Green, L. (2007). Delay discounting of cocaine by rhesus monkeys. Experimental and Clinical Psychopharmacology, 15, 238-244. doi:10.1037/10641297.15.3.238 